\title{
Further VLBA observations of SiO masers toward Mira variable stars ${ }^{\star}$
}

\author{
W. D. Cotton ${ }^{1}$, W. Vlemmings ${ }^{2}$, B. Mennesson ${ }^{3}$, G. Perrin ${ }^{4}$, V. Coudé du Foresto ${ }^{4}$, G. Chagnon ${ }^{4}$, P. J. Diamond ${ }^{2}$, \\ H. J. van Langevelde ${ }^{5,7}$, E. Bakker ${ }^{7, \star \star}$, S. Ridgway ${ }^{6}$, H. Mc Allister ${ }^{9}$, W. Traub ${ }^{3,8}$, and S. Ragland ${ }^{10}$ \\ 1 National Radio Astronomy Observatory ${ }^{\star \star \star}$, 520 Edgemont Road, Charlottesville, VA 22903-2475, USA \\ e-mail: bcotton@nrao.edu \\ 2 Jodrell Bank Observatory, University of Manchester, Macclesfield Cheshire, SK11 9DL, UK \\ 3 Jet Propulsion Laboratory, 4800 Oak Grove Drive, Pasadena, CA 91109, USA \\ ${ }^{4}$ DESPA, Observatoire de Paris, section de Meudon, 5 place Jules Janssen, 92190 Meudon, France \\ 5 Joint Institute for VLBI in Europe, Postbus 2, 7990 AA Dwingeloo, The Netherlands \\ ${ }^{6}$ NOAO, 950 N. Cherry Ave., PO Box 26732, Tucson, AZ. 85726, USA \\ 7 Leiden Observatory, PO Box 9513, 2300 RA Leiden, The Netherlands \\ 8 Harvard-Smithsonian Center for Astrophysics, 60 Garden Street, Cambridge, MA 02138, USA \\ 9 Georgia State University \\ 10 W. M. Keck Observatory, 65-1120 Mamalahoa Hwy, Kamuela, HI 96743, USA
}

Received 3 March 2006 / Accepted 7 June 2006

\section{ABSTRACT}

\begin{abstract}
We present the results of continued monitoring of the $\mathrm{SiO}$ masers at $7 \mathrm{~mm}$ wavelength in several of the Mira variable stars reported in Cotton et al. (2004, A\&A, 414, 275) $(o$ Ceti = Mira, U Orionis = U Ori, R Aquarii = R Aqr $)$ over a period of 16 months, extending the observations to several pulsation cycles. The observed size of the maser rings varied by $3-14 \%$ with time but show no clear correlation with pulsation phase. In all cases, the $\mathrm{SiO}$ masers appear just outside the dense molecular layer indicated by near-IR observations. Rotation (or large scale motion) is possibly detected in $o$ Ceti with a period of $89 \times \sin (i)$ years. We find linear polarization up to $\sim 60 \%$ and at several epochs predominantly tangentially ordered polarization vectors indicate a radial magnetic field direction. Jet-like features are examined in $o$ Ceti and R Aqr and in both cases, the magnetic field appears elongated with the masing structure. This suggests that the dynamic feature in the envelope is dragging the magnetic field or that the gas is constrained to follow magnetic field.
\end{abstract}

Key words. stars: atmospheres - stars: AGB and post-AGB - stars: variables: general - radio lines: stars - masers

\section{Introduction}

Mira variables are stars of a few solar masses which have reached the end of their lives and have moved to the Asymptotic Giant Branch (AGB) where the inner core of the star has collapsed and the outer envelope has become very extended and is in the process of losing its mass to the interstellar medium through pulsational instabilities. In the cool, extended envelopes of these stars, molecules form and condense into dust. In oxygen rich Miras, molecular masers appear, especially $\mathrm{SiO}, \mathrm{H}_{2} \mathrm{O}$ and $\mathrm{OH}$ at increasing distance from the photosphere (Reid \& Moran 1981). The SiO masers occur within a few stellar radii of the stellar surface between the hot molecular inner envelope and the cooler region at 3-5 $R_{\star}$ where the (silicate) dust forms; see Reid \& Menten (1997), Danchi et al. (1994).

Circumstellar $\mathrm{SiO}$ masers tend to occur in clumpy, partial rings centered on the central star (Diamond et al. 1994). This is presumed to be due to the longer paths through constant

^ Expanded views of Figs. 1-3 with and without polarization vectors are only available in electronic form at http://www . edpsciences.org

$\star \star$ Present Address: Eric Bakker, New Mexico Tech, Magdalena Ridge Observatory, Socorro, NM, 87801, USA.

$\star \star \star$ The National Radio Astronomy Observatory (NRAO) is operated by Associated Universities Inc., under cooperative agreement with the National Science Foundation. velocity gas needed for the masers to develop. Thus, these masers become markers for the layer in which the physical conditions necessary for the masers exist. The resolution available to VLBI observations is a very small fraction of the diameter of the ring; the limit on the accuracy of the measurement of the ring diameter is determined by the number and distribution of the maser spots. Much of the emission is confined in localized "spots" with lifetimes of a few months. Modeling of the dynamics of circumstellar $\mathrm{SiO}$ masers is developed in Humphreys et al. (1996) and Humphreys et al. (2002) which give plausible agreement with the observations.

VLBI observations of the $\mathrm{SiO}$ masers in this dynamically significant region provide a powerful probe. The frequent monitoring of the $\mathrm{SiO}$ masers in TX Cam by Diamond \& Kemball (2003) show complex motions of the masing gas. Both outward and inward motions are detected. Observations of TX Cam in both the $v=1, J=1-0$ and $v=2, J=1-0$ transitions of $\mathrm{SiO}$ at several epochs are discussed in Yi et al. (2005); masers in the two transitions show different distributions. Soria-Ruiz et al. (2004) have examined the distributions of masers in a number of lines of $\mathrm{SiO}$ in IRC +10011 and $\chi \mathrm{Cyg}$ and discuss the differences.

The polarization of circumstellar masers is a valuable probe of magnetic fields in circumstellar envelopes. The complex theory of maser polarization, and particularly that of the $\mathrm{SiO}$ masers, is described a large number of publications (e.g. Deguchi \& Watson 1990; Nedoluha \& Watson 1990; 
Table 1. Miras observed.

\begin{tabular}{lcrrrrrrr}
\hline \hline Star & Calibrator & $\begin{array}{r}\mathrm{UD}_{2.2 \mu}^{1} \\
(\mathrm{mas})\end{array}$ & $\begin{array}{r}\mathrm{UD}_{3.6 \mu}^{1}(\mathrm{mas}) \\
\hline o \text { Ceti }\end{array}$ & $\begin{array}{r}\text { Just }_{11 \mu}^{2} \\
(\mathrm{mas})\end{array}$ & $\begin{array}{r}\mathrm{Vel} \mathrm{A}^{3} \\
\left(\mathrm{~km} \mathrm{~s}^{-1}\right)\end{array}$ & $\begin{array}{r}\mathrm{Vel} \mathrm{B}^{4} \\
\left(\mathrm{~km} \mathrm{~s}^{-1}\right)\end{array}$ & Period & Sessions $^{5}$ \\
U Ori & $\mathrm{J} 0555+3944$ & $24.40(0.11)$ & $35.2(2.0)$ & 120 & 48.0 & 45.0 & 331.96 & ABCDEFGH \\
R Aqr & $\mathrm{J} 2334+0736$ & $15.59(0.06)$ & $28.8(0.1)$ & 160 & -34.0 & -40.0 & 368.30 & ABCDEFGH \\
\hline
\end{tabular}

${ }^{1} \mathrm{UD}=$ Uniform Diameter model fit (Mennesson et al. 2002). ${ }^{2}$ Fitted inner dust shell diameter from Danchi et al. $(1994) .{ }^{3} v=2, J=1-0$ transition of $\mathrm{SiO}$ at $42.8 \mathrm{GHz} .{ }^{4} v=1, J=1-0$ transition of $\mathrm{SiO}$ at $43.1 \mathrm{GHz} .{ }^{5} \mathrm{~A}, \mathrm{~B}, \mathrm{C}, \mathrm{D}$ from Cotton et al. (2004), E=25 Apr. 2003, $\mathrm{F}=26$ Oct. 2003, $\mathrm{G}=14$ May 2004, $\mathrm{H}=4$ Sep. 2004.

Elitzur 1991; Nedoluha \& Watson 1994; Elitzur 1996). VLBI Polarization observations of TX Cam by Kemball \& Diamond (1997) revealed tangentially ordered polarization vectors indicating a radial magnetic field direction and circular polarization indicating a magnetic field strength of several Gauss. Recent work by Herpin et al. (2006) indicates an average magnetic field of $3.5 \mathrm{G}$ in the $\mathrm{SiO}$ maser region of a large sample of evolved stars. Ordered magnetic fields were also found in the $\mathrm{H}_{2} \mathrm{O}$ and $\mathrm{OH}$ maser regions at larger distances from the star (Vlemmings et al. 2002; Etoka \& Diamond 2004; Vlemmings et al. 2005), where the $\mathrm{H}_{2} \mathrm{O}$ maser observations indicate that the magnetic field is strong and dynamically important. The influence of magnetic fields was recently also shown by the detection of the first magnetically collimated jet around an evolved star (Vlemmings et al. 2006).

Observations of $\mathrm{SiO}$ masers in a number of Miras at multiple epochs during a pulsation cycle were reported in Cotton et al. (2004). This paper presents the results obtained from continued monitoring of selected stars at an additional four epochs extending the time line covered to a period of 43 months covering several pulsation cycles.

\section{Observations and data reduction}

The new observations were obtained with the NRAO Very Long Baseline Array (VLBA) in four $10 \mathrm{~h}$ sessions on 25 Apr. 2003, 26 Oct. 2003, 14 May 2004, and 4 Sept. 2004. Two $4 \mathrm{MHz}$ wide channels in each right- and left-hand circular polarization were recorded at 42.820587 and $43.122027 \mathrm{GHz}$ to cover the $v=2, J=1-0$ and $v=1, J=1-0$ transitions of SiO. Two bit sampling was used in the recording. The correlations resulted in 128 channels in each of the combinations of right- and leftcircular polarization for each transition. A strong, nearby continuum source was observed before each star to serve as delay, bandpass and polarization calibrator. The observations are summarized in Table 1 which lists the stars, calibrators, central velocities and the dates observed; unless otherwise noted, the IR data are from Mennesson et al. (2002) and are uniform disk model diameter fits. The calibrators are all quasars with milliarcsecond accuracy positions; the Hipparchus (ESA 1997) positions of the stars, evaluated at the epoch of the observations, were used. Note: the center velocities were picked to center the spectra in the observing band and are not necessarily the stellar systemic velocities.

Calibration and processing follows the procedure described in Cotton et al. (2004) except as noted below. Linear polarization calibration was based on monitoring of the continuum calibrators from the VLA. The accuracy of the polarization angle calibration depends on the relative timing of the VLA calibrations and varies from epoch to epoch. In general, the calibration of polarization angle should be better than 10 degrees.
Due to geometric model errors used in the VLBA correlator, the calibration procedure failed to adequately align the images in the two transitions. The misalignment errors were typically a few milliarcseconds and were corrected by aligning features which appeared to be common in both transitions. This procedure has the potential to leave residual alignment errors.

The stars were observed with multiple snapshots which results in relatively limited uv-coverage given the large size and sometimes complex structure in a given channel. In order to minimize the effects of the limited uv-coverage, imaging was done using the Obit package task Imager which allows the automatic determination of CLEAN windows independently in each channel. CLEAN windows determined from Stokes $I$ images were used for CLEANing Stokes' $Q$ and $U$ images. This reduces the artifacts resulting from the poor uv-coverage while reducing the bias of setting the windows by hand as was done in Cotton et al. (2004).

As in Cotton et al. (2004), the "compressed" images combining the spectral channels incorporated only Stokes $I$ pixels determined significantly above the noise (from an rms cutoff) and above the level of artifacts from the limited dynamic range (from a fraction of the peak in the channel image) using Obit task Squish. Stokes' I images were compressed to 2-D by taking the maximum channel value in each pixel while for Stokes' $Q$ and $U$, the summed values were used. Since in a given pixel emission in only a limited range of velocity is seen, this procedure gives an adequate representation.

The maser spots occur in an extended, possibly non-circular, ring around the star. However, the number and distribution of spots is variable and generally insufficient to determine a model more complex that a circular diameter and ring thickness. Even with this approximation, joint analysis of the two transitions was needed to determine the center of the ring, the diameters were determined independently.

The diameters and widths of the $\mathrm{SiO}$ maser rings were determined from a moment analysis of compressed (2-D) Stokes $I$ images in both transitions using a direct parameter search which minimized the sum of the ring widths. This procedure, especially the estimated ring widths may be adversely affected by residual errors in the alignment of the images in the two transitions described above.

The velocity fields evident in the derived images are complex and change as individual maser spots brighten and fade. In order to look for evidence of systematic motion in the masing region, flux density weighted averages of the velocity were made in sectors around the maser ring with a width twice the second moment widths.

\section{Results}

The compressed total intensity images for each epoch and transition with superposed polarization "E" vectors are shown in 
W. D. Cotton et al.: Further VLBA observations of SiO masers toward Mira variable stars
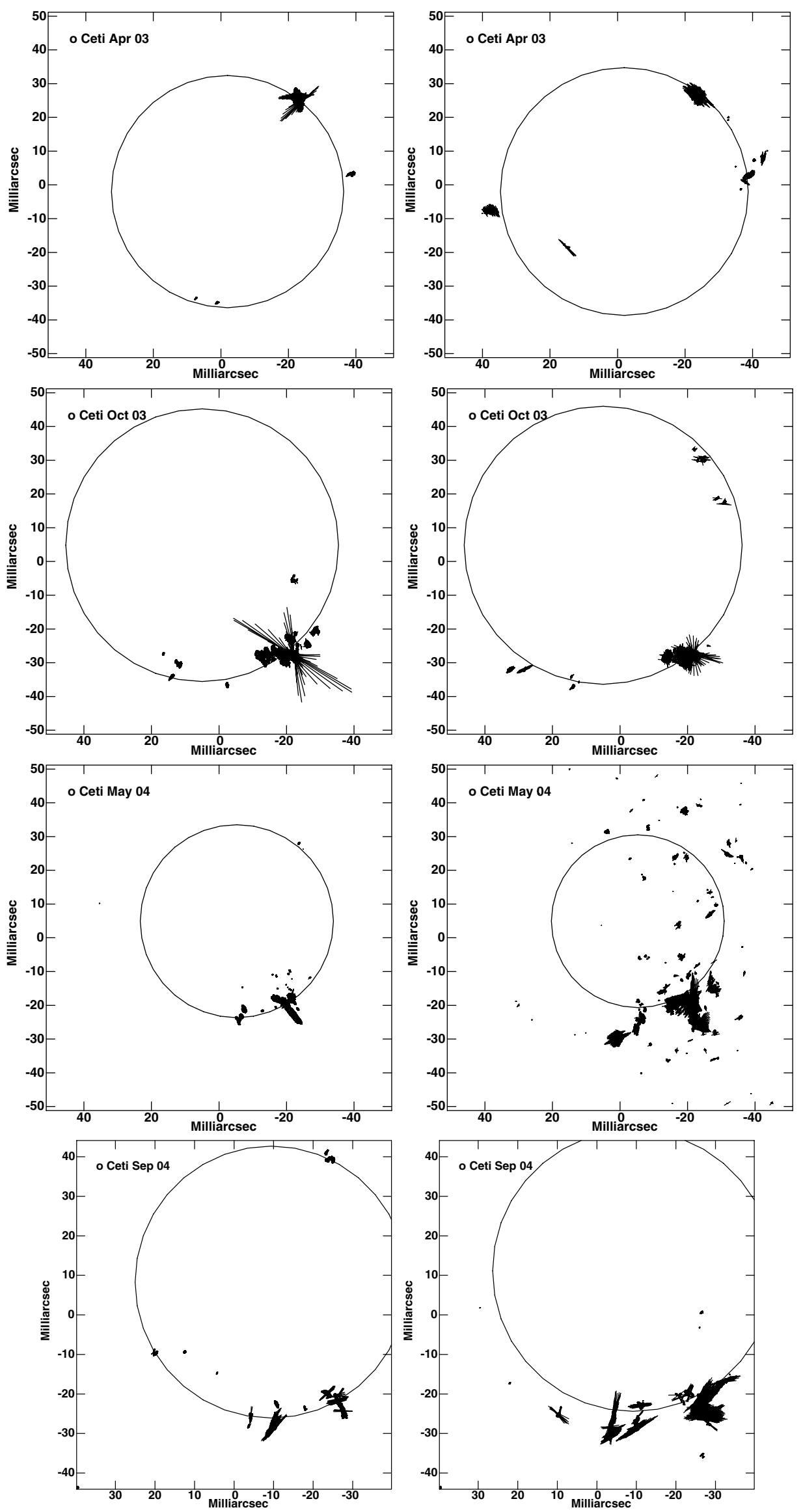

Fig. 1. $o$ Ceti (Mira). Left column is $v=2, J=1-0$ transition of $\mathrm{SiO}$ at $42.8 \mathrm{GHz}$, right column is $v=1, J=1-0$ transition of $\mathrm{SiO}$ at $43.1 \mathrm{GHz}$. Polarization "E" vectors are over-plotted on contours of total intensity. Circles show the fitted rings as given in Table 2 and are centered on the location determined to be the stellar position. 

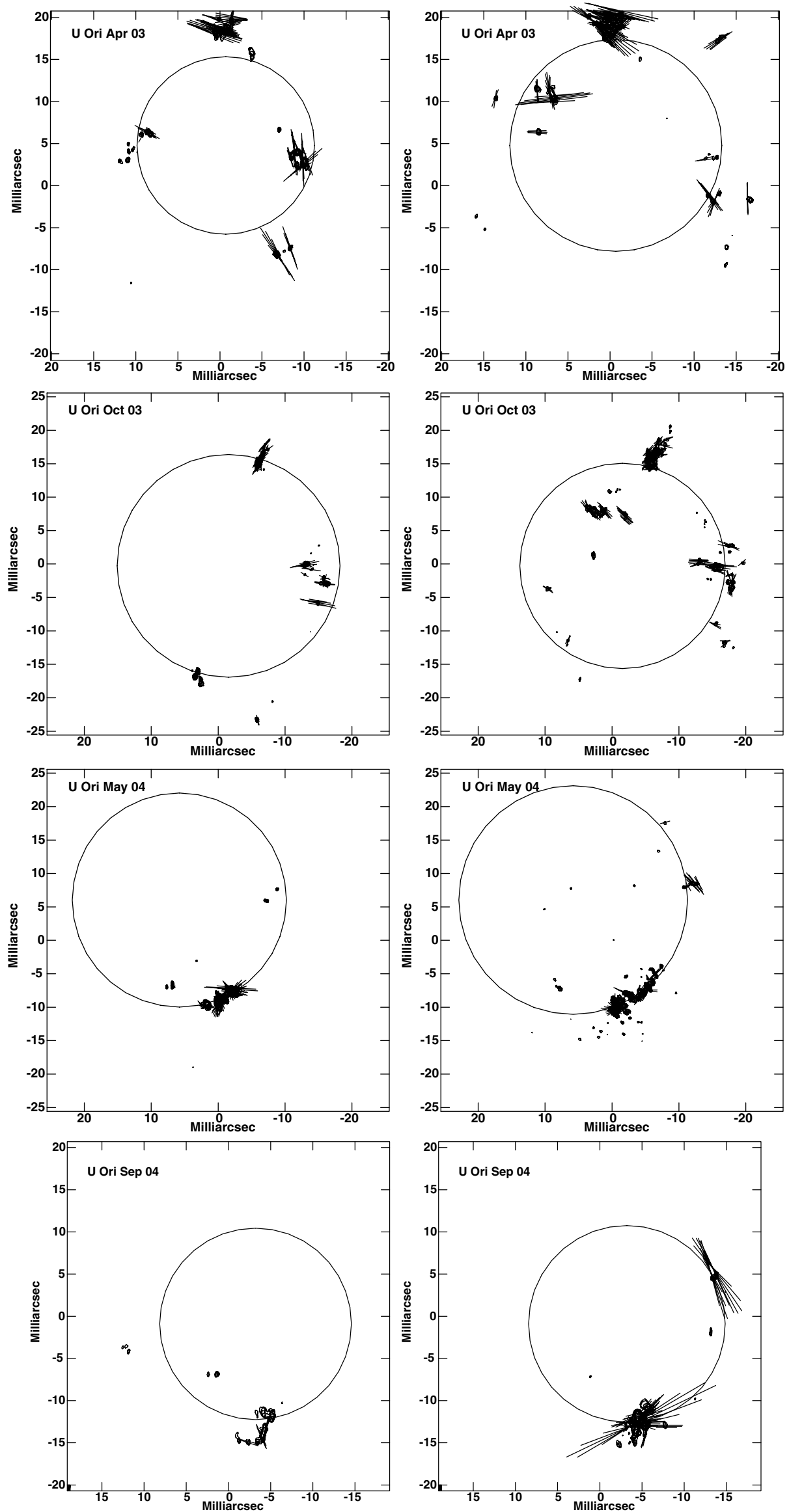

Fig. 2. U Ori. Left column is $v=2, J=1-0$ transition of $\mathrm{SiO}$ at $42.8 \mathrm{GHz}$, right column is $v=1, J=1-0$ transition of $\mathrm{SiO}$ at $43.1 \mathrm{GHz}$. Polarization "E" vectors are over-plotted on contours of total intensity. Circles show the fitted rings as given in Table 2 and are centered on the location determined to be the stellar position. 

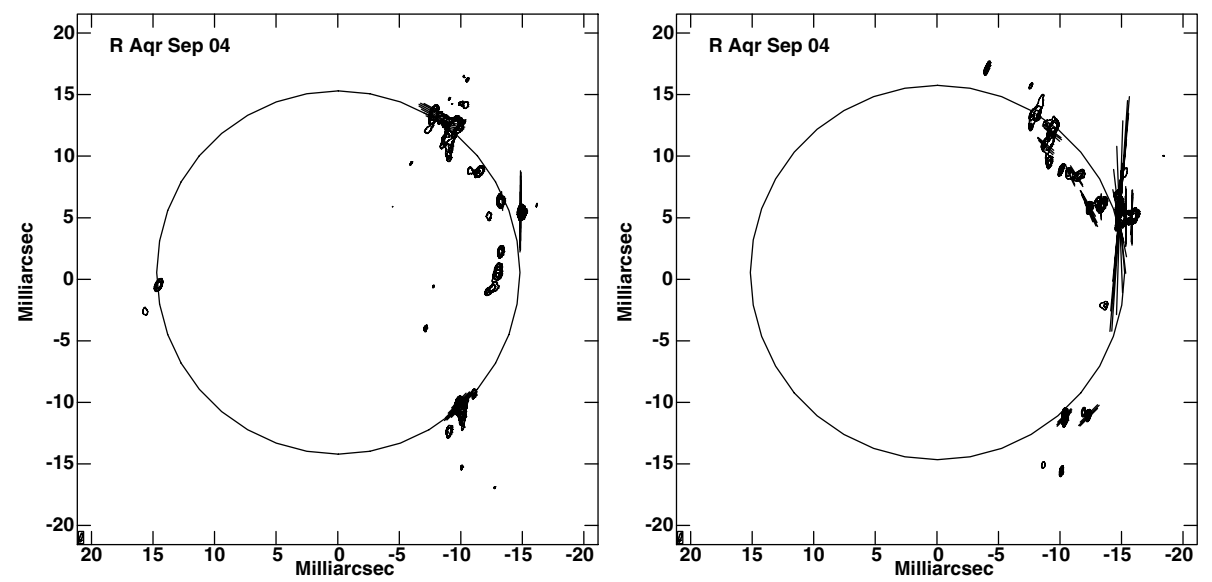

Fig. 3. R Aqr. Left column is $v=2, J=1-0$ transition of $\mathrm{SiO}$ at $42.8 \mathrm{GHz}$, right column is $v=1, J=1-0$ transition of $\mathrm{SiO}$ at $43.1 \mathrm{GHz}$. Polarization "E" vectors are over-plotted on contours of total intensity. Circles show the fitted rings as given in Table 2 and are centered on the location determined to be the stellar position.

Table 2. SiO ring diameters.

\begin{tabular}{lrrrrrrrr}
\hline \hline & Apr. 03 (E) & & Oct. 03 (F) & & May 04 (G) & \multicolumn{3}{c}{ Sep. 04 (H) } \\
Source & Diam (mas) & Width & Diam (mas) & Width & Diam (mas) & Width & Diam (mas) & Width \\
\hline$o$ Ceti [1] & 73.4 & 3.9 & 82.4 & 2.6 & 61.2 & 3.4 & 71.1 & 2.6 \\
$o$ Ceti [2] & 68.8 & 1.3 & 80.8 & 2.5 & 57.2 & 2.2 & 68.8 & 2.3 \\
U Ori [1] & 25.2 & 2.9 & 30.7 & 6.6 & 34.1 & 0.6 & 23.3 & 1.4 \\
U Ori [2] & 21.1 & 2.4 & 33.2 & 7.3 & 32.0 & 0.6 & 22.7 & 2.1 \\
R Aqr [1] & & & & & & & 30.4 & 0.9 \\
R Aqr [2] & & & & & & & 29.5 & 0.6 \\
\hline
\end{tabular}

Notes: ${ }^{1}[1]$ denotes $v=1, J=1-0$ transition of $\mathrm{SiO}$ at $43.1 \mathrm{GHz} ;{ }^{2}[2]$ denotes $v=2, J=1-0$ transition of $\mathrm{SiO}$ at $42.8 \mathrm{GHz}$.

Figs. 1-3. (Expanded views of these figures with and without polarization vectors are available in electronic form.) The fitted ring diameters and widths are shown in Table 2 and in Figs. 4-6 as well as in Figs. 1-3. Each row in Table 2 corresponds to a given star and transition and the measured ring diameter and width for each session are given in separate columns.

The VLBA array used for these observations has no baselines short enough that the $\mathrm{SiO}$ emission in the stars observed is not strongly resolved. Thus, only the brightest regions of emission are visible and typically only a fraction of the single dish emission is represented in the images. Furthermore, the calibration technique results in relatively poor absolute calibration. An examination of the correlation between the IR flux and maser emission as was done in Pardo et al. (2004) is not possible with the images shown in Figs. 1-3. A discussion of the relationship between interferometric and single dish measurements is given below.

\section{Discussion}

\subsection{Maser ring diameters}

Figures 4-6 indicate that the diameter of the ring in the $v=2$, $J=1-0$ transition is always smaller than that in the $v=1, J=$ 1-0 transition. Yi et al. (2005) report similar results for TX Cam and by Desmurs et al. (2000) in TX Cam and IRC+10011. The shock pumped model of Humphreys et al. (1996) predicts that the $v=2, J=1-0$ transition should produce a smaller diameter ring than the $v=1, J=1-0$ transition. The models of Humphreys et al. (2002) in which variations in the $\mathrm{SiO}$ maser emission is due to shocks predict an rms variation in the measured ring diameter of $6 \%$ over a luminosity cycle. The apparent

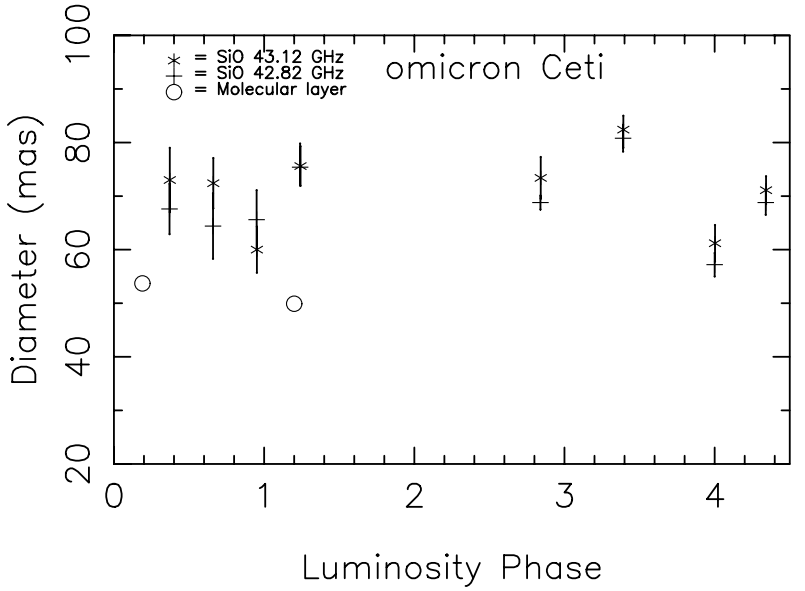

Fig. 4. The SiO and IR diameters as a function of luminosity phase for $o$ Ceti (Mira). Vertical bars on the maser symbols represent the width of the ring as determined from the second moment about the diameter of the ring. The "molecular layer" is the derived value of the diameter of the molecular layer from Perrin et al. (2004), the uncertainty is less than the symbol size.

size of the ring decreases when the shock from a new stellar pulsation pushes new material into the $\mathrm{SiO}$ maser zone. Since the pulsation period of the stars presented here is approximately a year and the span of the observations approximately three and a half year, reasonable, if sparse, sampling was made over several luminosity cycles. The mean and rms variations in the fitted ring diameters for each of the stars observed in all four sessions are given in Table 3 for each of the two transitions observed. 


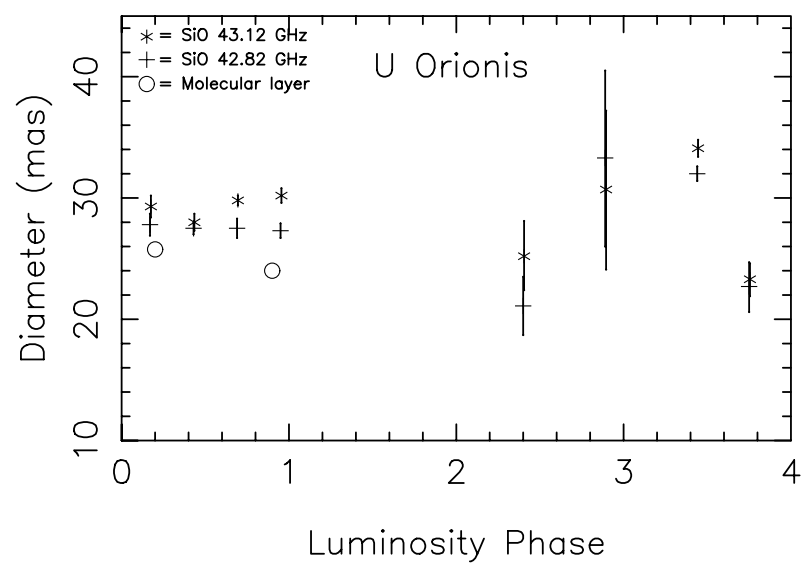

Fig. 5. As in Fig. 4 but for U Ori.

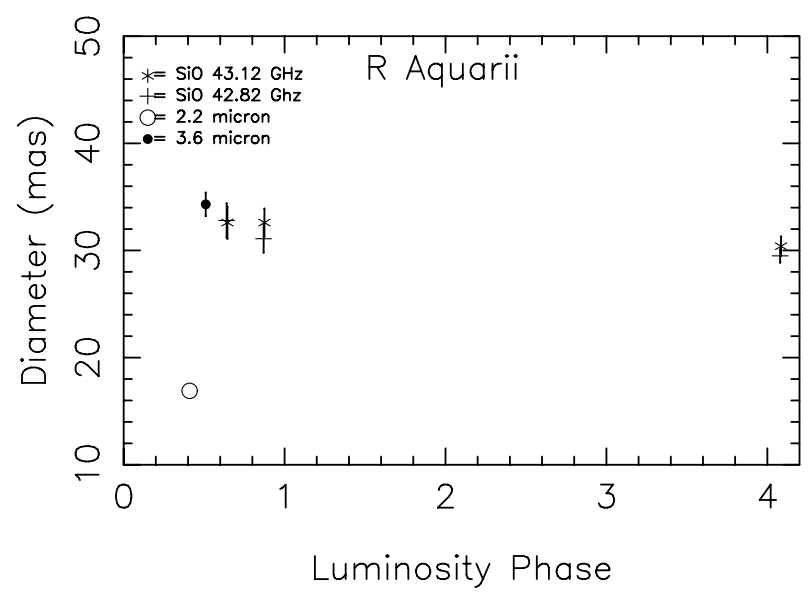

Fig. 6. As in Fig. 4 but for R Aqr and with IR diameters from Mennesson et al. (2002). The vertical bar on the $3.6 \mu \mathrm{m}$ symbol indicates the error bar.

Since the maser rings are frequently sparsely populated with maser spots, the accuracy of the estimation of the diameter of the ring is limited by the number and placements of the spots. All of the stars given in Table 3 show smooth variations in the ring diameter and the two transitions track each other. All are roughly in agreement with the Humphreys et al. (2002) rms variation of $6 \%$. In the earlier observations of Cotton et al. (2004), U Ori was nearly constant in size but the more recent observations presented here show more variability.

The variation of the ring diameter with luminosity phase shown in Figs. 4-6 varies dramatically from star to star and cycle to cycle in a given star. This disagrees with the models of (Humphreys et al. 2002) which predicts that the behavior of the ring diameters with luminosity phase should be similar for all stars. Some of this disagreement may be the result of the difficulty determining the ring diameter in some of the observations.

Figures 4 and 5 also show the results of model fitting to narrowband near-IR interferometric data by Perrin et al. (2004). In both these cases, the $\mathrm{SiO}$ masers appear to come from a region just outside the dense molecular region. The $3.6 \mu \mathrm{m}$ diameter shown in Fig. 6 is thought to be dominated by the molecular layer (Mennesson et al. 2002) and is comparable to the size of the $\mathrm{SiO}$ maser ring.
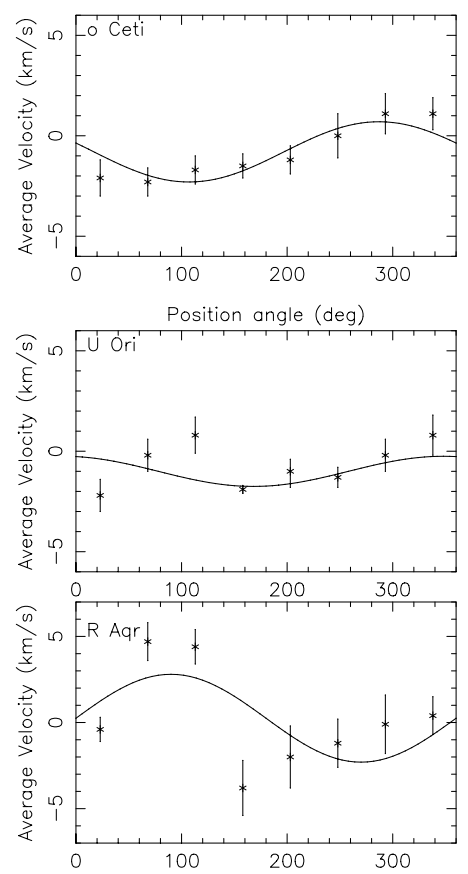

Fig. 7. The sector averaged velocities over epoch and transition. The solid line represents a model fit (see text).

\subsection{Motion of the envelope}

Since the detectable masers are largely in the tangent through the $\mathrm{SiO}$ maser layer, all radial velocities, as seen by us, will generally be azimuthal to the star. Thus, the variation in velocity of maser spots in location in the ring and in time is due either to systematic motions, such as rotation of the star or turbulent motions in the atmosphere. Stars with close companions could have systematic motions induced in the envelope due to tidal interactions. There is some correlation between the velocities observed in the two transitions, as would be expected if the masers arise in the same regions. There are large variations in the sector averages velocities in time, even in sectors with a reasonable flux density of masers. These fluctuations of a few $\mathrm{km} \mathrm{s}^{-1}$ are undoubtedly due to changes in the large scale bulk motions in the stellar atmosphere.

However, with up to 8 epochs and using both transitions, random motions will tend to average out and it is possible to constrain the systematic motions. We will interpret any such systematic motion as rotation, although noting that stellar rotation in such large objects is difficult to explain. A "rotation" velocity (more properly velocity $\times \sin (i)$ where $i$ is the inclination of the rotation pole to the line of sight) was fitted using a least squares fitting procedure on the sector average velocities. The fitted parameters were the rotation velocity at the location of the maser ring (Rotn), the position angle of the pole (PA) and an offset of the systemic velocity from the assumed center velocity (Offset). Errors were estimated from the change in the value needed to double the $\chi^{2}$ of the fit. The results are given in Table 4 and Fig. 7 with the model values shown as the solid lines in Fig. 7. The $\chi^{2}$ values given in Table 4 are per degree of freedom. Error estimates are given in parentheses following the value.

\subsection{Linear polarization}

The vectors in Figs. 1-3 are proportional to the linearly polarized intensity, and their orientation defines the plane of the electric field. We find that for all three sources the fractional linear 
Table 3. SiO ring diameter variations.

\begin{tabular}{lrrrrrrrr}
\hline \hline Star & $\begin{array}{r}\text { Mean } \\
(\mathrm{mas})\end{array}$ & $\begin{array}{r}\text { Mean } \\
\left(2 R_{2.2 \mu}\right)\end{array}$ & $\begin{array}{r}\mathrm{rms}^{1} \\
(\mathrm{mas})\end{array}$ & $\begin{array}{r}\text { Percent }^{1} \\
(\%)\end{array}$ & $\begin{array}{r}\text { Mean }^{2} \\
(\mathrm{mas})\end{array}$ & $\begin{array}{r}\text { Mean } \\
\left(2 R_{2.2 \mu}\right)\end{array}$ & $\begin{array}{r}\mathrm{rms}^{2} \\
(\%)\end{array}$ & Percent $^{2}$ \\
\hline$o$ Ceti & 71.1 & 2.92 & 6.9 & 10 & 68.6 & 2.81 & 6.6 & 10 \\
U Ori & 28.8 & 1.85 & 3.1 & 11 & 27.4 & 1.76 & 3.8 & 14 \\
R Aqr & 31.9 & 1.89 & 1.0 & 3 & 31.1 & 1.84 & 1.3 & 4 \\
\hline
\end{tabular}

${ }^{1} v=1, J=1-0$ transition of $\mathrm{SiO}$ at $43.1 \mathrm{GHz} .{ }^{2} v=2, J=1-0$ transition of $\mathrm{SiO}$ at $42.8 \mathrm{GHz}$.

Table 4. Envelope rotation fits.

\begin{tabular}{lrrrr}
\hline \hline Star & $\begin{array}{r}\text { Rotn } \\
\mathrm{km} \mathrm{s}^{-1}\end{array}$ & $\begin{array}{r}\text { PA } \\
\circ\end{array}$ & $\begin{array}{r}\text { Offset } \\
\mathrm{km} \mathrm{s}^{-1}\end{array}$ & $\chi^{2}$ \\
\hline$o$ Ceti & $1.50(0.90)$ & $197(36)$ & $-0.80(0.65)$ & 1.01 \\
U Ori & $0.75(0.75)$ & $258(90)$ & $-1.00(0.65)$ & 3.03 \\
R Aqr & $2.55(2.50)$ & $0(72)$ & $0.25(2.05)$ & 5.09 \\
\hline
\end{tabular}

polarization varies strongly over time, with the maximum linear polarization fraction changing from $m_{1} \sim 15 \%$ to $m_{1} \sim 65 \%$ at different epochs. All three sources and both maser transitions show a similar spread in $m_{1}$. Similar to the observations of TX Cam by Kemball \& Diamond (1997) and TX Cam and IRC +10011 by Desmurs et al. (2000), the polarization position angles at significant portions of the inner shell boundary are tangential. However, for some of the maser features of both $o$ Ceti and $\mathrm{U}$ Ori, the polarization angles are radial instead of tangential. The relation between the polarization angle and the direction of the magnetic field is complex. In Goldreich et al. (1973), it is shown that the magnetic field direction is either parallel or perpendicular to the polarization vectors, depending on the angle $\theta$ between the line-of-sight and the magnetic field. When $\theta<\theta_{\text {crit }} \approx 55^{\circ}$, the polarization vectors are parallel to the magnetic field and when $\theta>\theta_{\text {crit }}$ they are perpendicular. However, this relation is only valid when the Zeeman frequency shift under the influence of the magnetic field $g \Omega$ is much larger than the rate of stimulated emission $R$. Otherwise, as shown in Nedoluha \& Watson (1990), the relation between polarization angle and magnetic field direction is dependent on the maser intensity. For the $\mathrm{SiO}$ masers in a magnetic field of $1-10 \mathrm{G}$, $10^{3}<g \Omega<10^{4}$. This indicates that $g \Omega \gg R$ is satisfied for all but the strongest of the masers. For those masers with fluxes $\sim 100 \mathrm{Jy}$, Nedoluha \& Watson (1990) find that the polarization angle and magnetic field direction can be offset by up to $\sim 20^{\circ}$. Thus, as argued in Kemball \& Diamond (1997), the predominantly tangential polarization vectors indicate a mostly radial magnetic field. The radial polarization vectors for some of the features of U Ori and $o$ Ceti possibly indicate that for those masers $\theta$ is close to $\theta_{\text {crit }}$.

\subsection{Jet-like features}

In a number of the maser images presented here and in Cotton et al. (2004) there are extended, linear features aligned with the direction to the photosphere. These frequently have similar morphologies in both the transitions observed with similar runs of velocity along their lengths. These features are notable in $\mathrm{R}$ Aqr in January 2001, and $o$ Ceti in August 2001 and May 2004. These features are shown in detail in Figs. 8-10. These features will be referred to as jet-like features in the following. These extended features may be fairly rare as the cases listed above are the only ones in which plausibly coherent features as opposed to a collection of individual maser spots are seen.
The jet-like features in $o$ Ceti differ from those in R Aqr in morphology. However, in both sources there is a strong tendency for the polarization vectors to the perpendicular to the direction of the feature. As the polarization vectors are mostly perpendicular to the magnetic field, this indicates radial magnetic field lines along the feature, which strengthens the impression that these are physical features rather than chance alignments of maser spots.

Another feature of these linear structures is the systematic nature of the radial (as seen by us) velocity variations. Since the observations are too infrequent to measure proper motions in these features, only the radial velocities are known. In addition, since these features are near the masing ring, these motions will be predominantly non-radial with respect to the star so do not represent simple acceleration of the masing material away from the star. The apparent alignment of the magnetic field with these features could be due either to the magnetic field being stretched along the features by the ionized component of the gas or the gas being constrained by its ionized component to move along field lines, depending on the relative energy density of the magnetic field. $\mathrm{SiO}$ maser observations (Herpin et al. 2003, 2006) and $\mathrm{H}_{2} \mathrm{O}$ maser observations (Vlemmings et al. 2005, 2006) suggest that magnetic fields are likely dynamically important in which case the maser jet-like features are following the magnetic field.

The jet-like feature shown of R Aqr in Fig. 8 was discussed in Cotton et al. (2004, See their Fig. 14) in which there is a similar feature on the opposite side of the star aligned along the apparent rotational equator of the star. The velocity fields of these features were consistent with being part of the stellar rotation. It is not clear that the linear features in $o$ Ceti are similar to those in R Aqr, both of the sets of linear features are in the southern hemisphere and may be illustrating the general rotation in the stellar envelope. In both cases, the radial velocity is highest at the end furthest from the star.

\subsection{Interferometric and single dish observations}

VLBI interferometetric and single dish measurements of circumstellar masers sample very different size scales; submilliarcsecond and arcminute scales respectively. This difference in scales can cause difficulty in the comparison of results for these two techniques. A further complication is the difference in calibration techniques, for single dish measurements, the objective is to obtain accurate total power measurements whereas for VLBI imaging, the objective is generally to minimize the imaging artifacts. The amplitude calibration scheme used for the data presented here consists of a number of steps:

1. system temperature and gain calibration. The "system temperature" (background level) for each receiver is monitored during the observations and together with standard sensitivity values are used for the initial calibration. Estimates of the atmospheric opacity are made from the system temperature measurements and applied as well; 

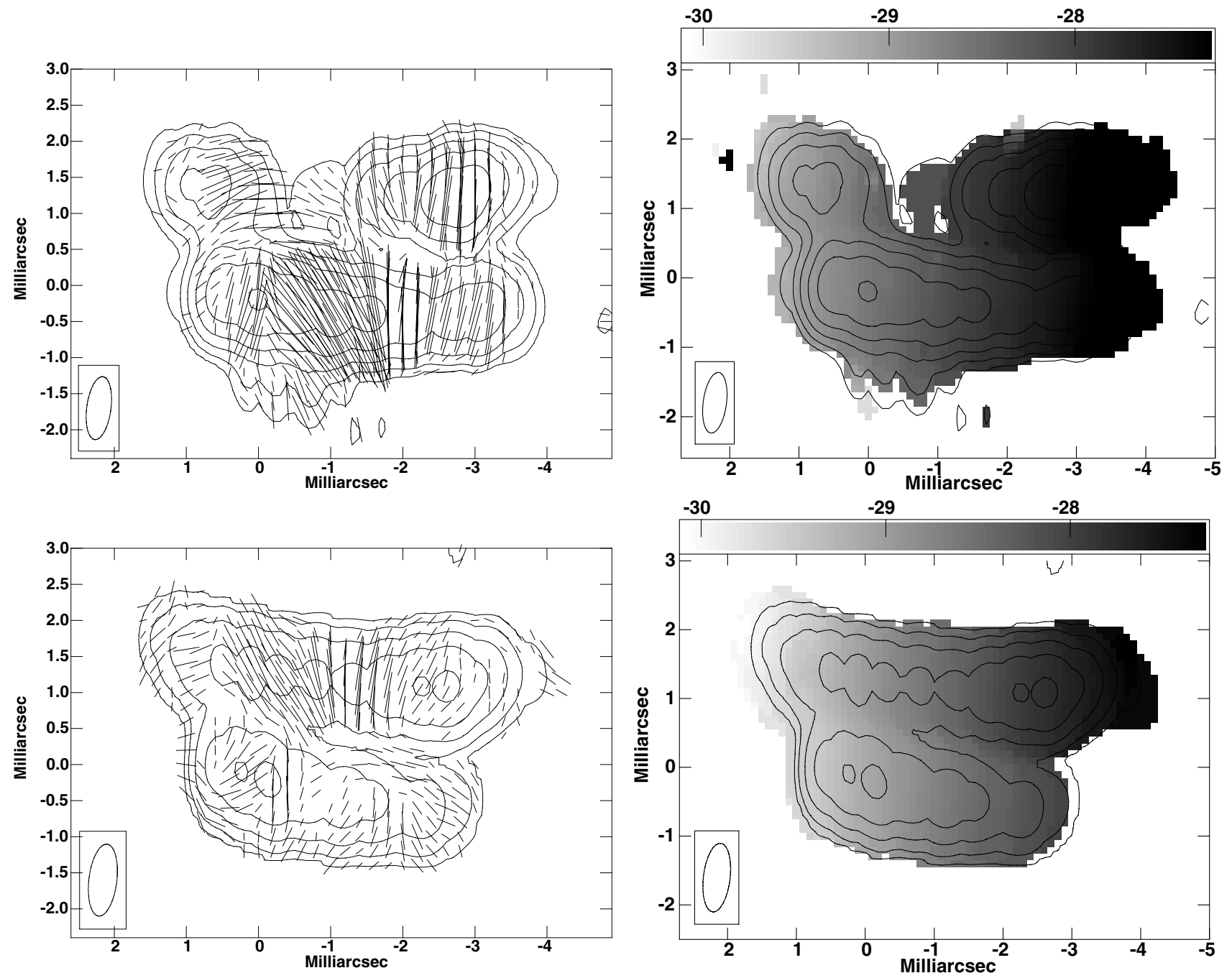

Fig. 8. Top: jet-like feature in R Aqr, January 2001 in the $\mathrm{SiO} v=2, J=1-0$ transition. On the left is a contour plot of peak (in velocity) Stokes $I$ with superposed vectors showing the direction and relative strengths of the polarization "E" vectors. Contours are separated by factors of $\sqrt{2}$. The ellipse in the lower left corner gives the resolution. On the right are contours in peak Stokes $I$ with a gray scale giving the Stokes' $I$ weighted average velocity in $\mathrm{km} \mathrm{s}^{-1}$. The velocity scale is given on the wedge at the top. Contours are separated by factors of 2 . The star center is to the left. Data from Cotton et al. (2004). Bottom: like top, but $v=1, J=1-0$ transition.

2. corrections from continuum calibrators. The system temperature and standard gain calibration leaves gain errors of typically a few 10s of percent which depend on source and time. These are largely due to antenna pointing errors and unmodeled atmospheric opacity variations. Averages of the gain corrections from self-calibration of several of the stronger continuum sources are used to refine the amplitude calibration;

3. template fitting. The ultimate amplitude calibration is derived from the autocorrelation (single dish) spectra obtained with the interferometric spectra and calibrated as described above. A template spectrum is picked from a time segment of autocorrelation data from one of the antenna with the best sensitivity (usually Mauna Kea) and gain factors for all other autocorrelation spectra are fitted to match the template spectrum. This process removes the effects of antenna mispointing and unmodeled atmospheric opacity variations but at the cost of compromising the absolute calibration. The autocorrelation data are not sensitive to long-term phase noise so coherence loss is not included in this calibration.

The template fitting technique results in better image quality but the absolute calibration can be off by 10s of percent, largely depending on the weather. Thus, the fluxes densities derived in the interferometric images are not easily comparable to properly calibrated single dish measurements.

However, the interferometric spectra can be compared to the template spectra which share most of the same systematic errors. This comparison will show the fraction of the single dish emission represented in the image. A comparison of the total spectrum and the spectra derived from the channel images is given in Figs. 11-13. These figures indicate that the VLBI images contain from a few 10s of percent to nearly all of the single dish flux density. The exact fraction of flux recovered in the images varies for different spectral channels across the spectrum. The figures also show the highly variable nature of the spectra. The data presented here are sampled too coarsely in time for a useful comparison with infrared flux variations.

\section{6. o Ceti (Mira)}

$o$ Ceti was observed in all four sessions reported in this paper as well as all four sessions in Cotton et al. (2004); see Figs. 1 and 4. The fitted diameter of the ring in May 2004 is poorly constrained by the distribution of maser spots and undoubtedly 

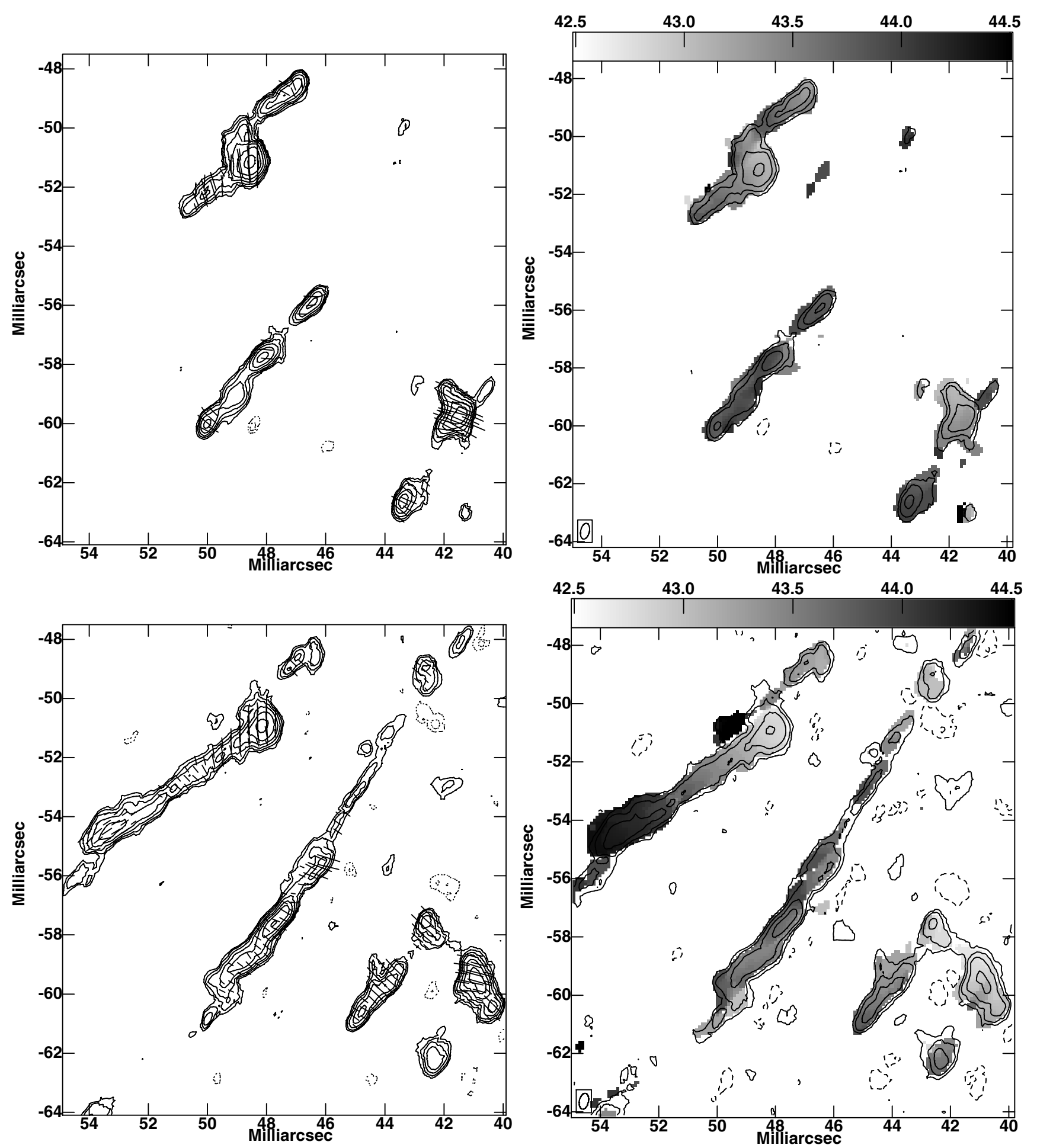

Fig. 9. Top: detailed view of jet-like feature in $o$ Ceti in August 2001 in the $\mathrm{SiO} v=2, J=1-0$ transition. On the left is a contour plot of peak (in velocity) Stokes $I$ with superposed vectors showing the direction and relative strengths of the polarization "E" vectors. Contours are separated by factors of $\sqrt{2}$. On the right are contours in peak Stokes $I$ with a gray scale giving the Stokes' $I$ weighted average velocity in $\mathrm{km} \mathrm{s}^{-1}$. The velocity scale is given on the wedge at the top. Contours are separated by factors of 2. Data from Cotton et al. (2004). Star center is to the upper right. The ellipse in the lower left corner gives the resolution. Bottom: like top, but $v=1, J=1-0$ transition. The length of the polarization vectors are reduced by a factor of three relative to the top figure.

accounts for the unusually small ring size fitted. Model fitting of the molecular layer by Perrin et al. (2004) gives a diameter around 50 mas putting the $\mathrm{SiO}$ masers at the outer edge of this region.

Figure 7 and the model fit shown in Table 4 provide some evidence of a systematic movement of the molecular envelope. The $\chi^{2}$ of the sector average velocities assuming no rotation is 29.6 , which for 7 degrees of freedom has a probability of occurrence of $<0.1 \%$. The fit including rotation given in Table 4 and shown in Fig. 7 is quite good $\left(\chi^{2}\right.$ per degree of freedom $\left.=1\right)$. This would give the rotation velocity (times $\sin (i)$ ) at a radius of 35 mas of $1.5 \mathrm{~km} \mathrm{~s}^{-1}$, or, for $i=90$, a rotation period of 89 years at an 

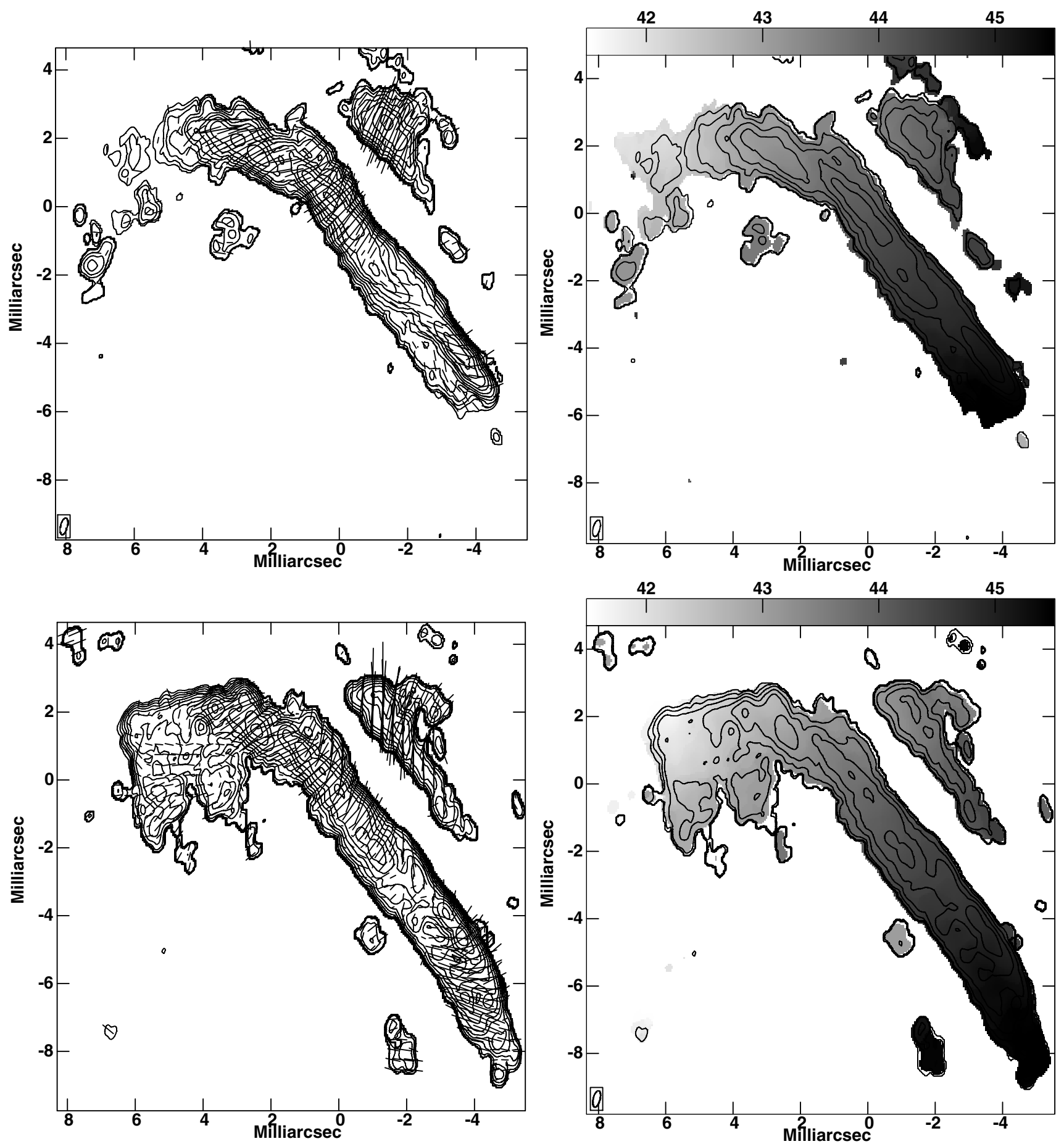

Fig. 10. Top: detailed view of jet-like feature in $o$ Ceti in May 2004 in the $\mathrm{SiO} v=2, J=1-0$ transition from Fig. 1. On the left is a contour plot of peak (in velocity) Stokes $I$ with superposed vectors showing the direction and relative strengths of the polarization "E" vectors. Contours are separated by factors of $\sqrt{2}$. On the right are contours in peak Stokes $I$ with a gray scale giving the Stokes' $I$ weighted average velocity in km s ${ }^{-1}$. The velocity scale is given on the wedge at the top. Contours are separated by factors of 2 . Star center is to the upper left. The ellipse in the lower left corner gives the resolution. Bottom: like top, but $v=1, J=1-0$ transition. The length of the polarization vectors are reduced by a factor of three relative to the top figure.

assumed distance of $128 \mathrm{pc} . o$ Ceti has a close companion which could be inducing systematic motion in the envelope.

The systemic velocity derived from this fitting is $45.7 \pm$ $0.7 \mathrm{~km} \mathrm{~s}^{-1}$. This is to be compared with the value of $46.4 \pm 0.3$ from Bowers \& Knapp (1988).

Cotton et al. (2004) speculated that the jet-like features in R Aqr in January 2001 were part of a rotating molecular disk.
This seems to be less likely the case for the jet-like features seem in $o$ Ceti in August 2001 and May 2004 as they are nearly orthogonal to each other. In addition, the admittedly weak evidence for rotation seen in Fig. 7 would put the rotational pole near the orientation of the jet-like feature seen in May 2004.

The polarization vectors across the jet-like feature are predominantly perpendicular to the feature which, as discussed 

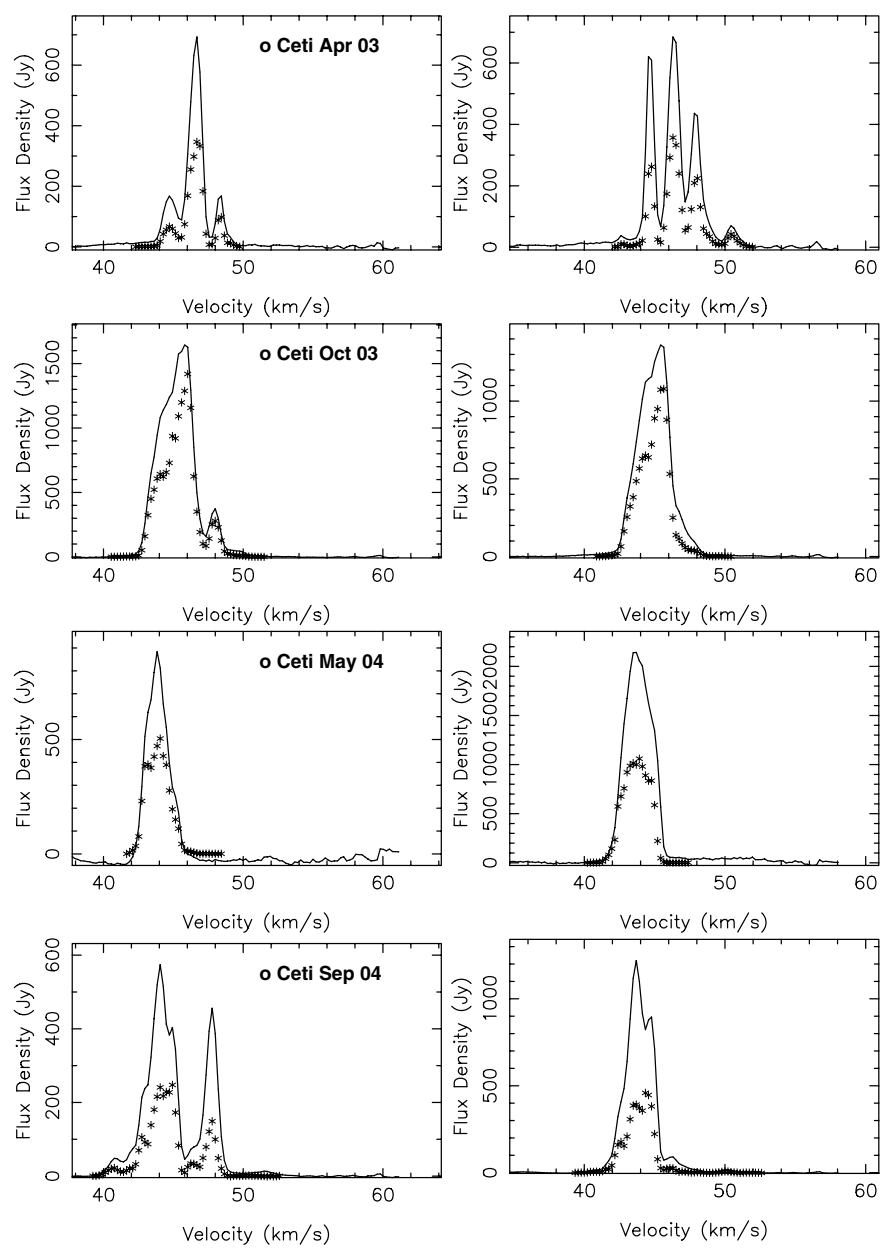

Fig. 11. Spectra of $o$ Ceti (Mira). Left column is $v=2, J=1-0$ transition of $\mathrm{SiO}$ at $42.8 \mathrm{GHz}$, right column is $v=1, J=1-0$ transition of $\mathrm{SiO}$ at $43.1 \mathrm{GHz}$. Solid lines are the total spectrum and stars indicate the flux density in the channel images.

above, likely indicate a magnetic field along the feature. A striking difference between the features observed in August 2001 compared to those observed in May 2004 is the fractional linear polarization. The features from August 2001 in Fig. 9 have $m_{1}$ between 15-20\%, however, those in May 2004, in Fig. 10, have $m_{1}$ up to $60 \%$. For both epochs $m_{1}$ of the $v=1$ transition is larger than that of the $v=0$ transition by $\approx 10 \%$. As the fractional linear polarization depends on both the angle between the line-of-sight and the magnetic field as well as the maser saturation level, the larger $m_{1}$ of the features from May 2004 might indicate that they are more saturated. The alignment of the polarization, and likely the magnetic field, with the jet-like features in $o$ Ceti suggests that these are dynamic features of the envelope rather than part of a general rotation of the envelope.

\subsection{U Ori}

This star was observed in all four sessions reported in this paper and all four in Cotton et al. (2004). In October 2003, the maser spot distribution does not appear to be well approximated by a ring, thus the fitted ring diameters showing the $v=2, J=1-0$ transition ring larger than the $v=1$ transition is likely spurious. The observations from Cotton et al. (2004) showed very little variation in the ring diameter but this trend is not seen in the more recent data. As for $o$ Ceti, model fitting of the molecular
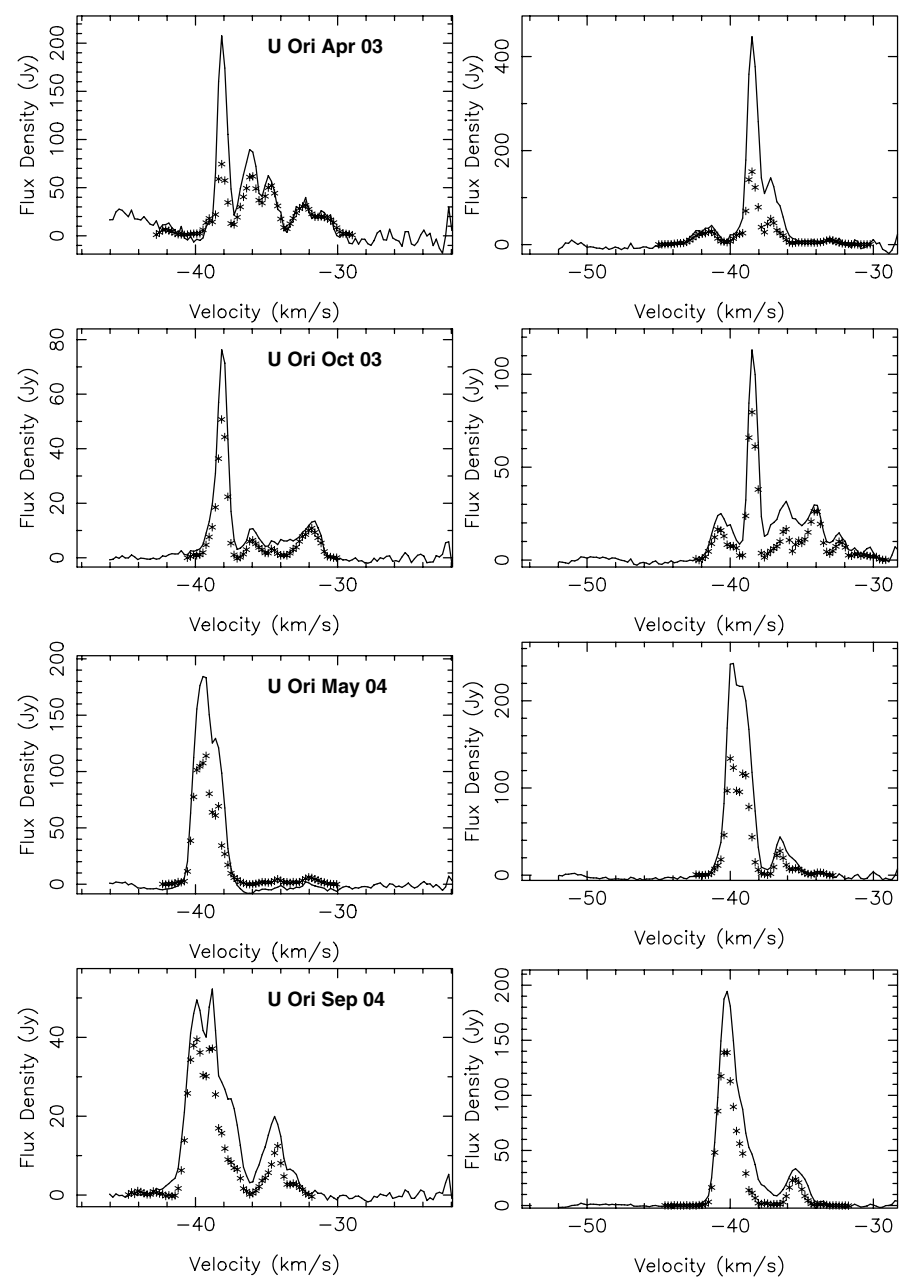

Fig. 12. Similar to Fig. 11 but for U Ori.
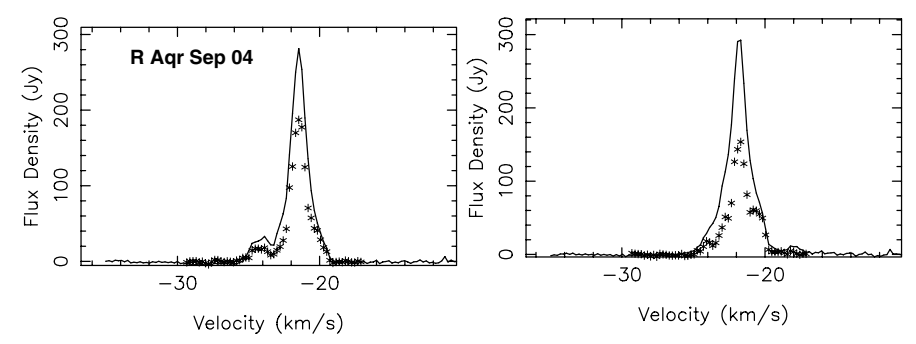

Fig. 13. Similar to Fig. 11 but for R Aqr.

layer by Perrin et al. (2004) puts the $\mathrm{SiO}$ masers at the outer edge of this region.

The evidence for a systematic motion in Fig. 7 and Table 4 is not convincing. The $\chi^{2}$ of the sector average velocities about a total average is 16.8, which for 7 degrees of freedom has a probability of chance occurrence of $2 \%$. However, the $\chi^{2}$ per degree of freedom is 2.4 which is better than the model fit value of 3.0 .

\section{8. $R$ Aqr}

This star is in a symbiotic system and exhibits a jet seen both optically and in the radio (Hollis et al. 1999) apparently emanating from an accretion disk around the hot companion star. Differential rotation in R Aqr at epoch 2001.0 is claimed in Hollis et al. (2000), Hollis et al. (2001) and Cotton et al. (2004). 
Hollis et al. (2001) gives a position angle of the rotation pole of $\approx-30 / 150^{\circ}$ and Cotton et al. (2004) give $\approx-10 / 170^{\circ}$ and a velocity at the maser ring of $3.9 \mathrm{~km} \mathrm{~s}^{-1}$ based on the January 2001 observations alone. The analysis of all data shown in Fig. 7 and Table 4 give a pole $\approx 0 / 180^{\circ}$ and a somewhat lower velocity of $2.6 \mathrm{~km} \mathrm{~s}^{-1}$. However, R Aqr was only observed at three epochs so the statistical analysis is not very accurate and the model fit is poor, $\chi^{2}$ per degree of freedom of 5.1. The bulk of the evidence for stellar rotation remains with the 2001 data. Hollis et al. (2000) suggest that the symmetric emission seen in 2001 and several years prior to this were due to the passage of the companion of R Aqr through its envelope circa 1996.

\section{Conclusions}

We present new total intensity and linear polarization VLBA observations of the $v=2$ and $v=1 J=1-0$ maser transitions of $\mathrm{SiO}$ at 42.8 and $43.1 \mathrm{GHz}$, extending the observations of Cotton et al. (2004) for a number of Mira variables to a total of eight epochs covering 43 months.

All three stars examined in this paper show variations in the $\mathrm{SiO}$ ring diameter with a range of rms variations (3-14\%) consistent with the models of Humphreys et al. (2002). The relationship between ring diameter and luminosity phase was not consistent among different stars or between pulsation cycles in the same star; this differs from the model predictions. In all three cases studied here the $\mathrm{SiO}$ masers appear to form just outside of the dense molecular region of the stellar atmosphere as determined by Perrin et al. (2004) and Mennesson et al. (2002).

A combined analysis of the kinematic data on $o$ Ceti suggests systematic motions which, if interpreted as a stellar rotation, gives a period of $89 \times \sin (i)$ years. Rotation has previously been claimed for R Aqr (Hollis et al. 2000; Hollis et al. 2001; Cotton et al. 2004). Both of these stars are in binary systems so it is possible that the motion of the envelope is the result of the secondary. The secondary of R Aqr passed through its envelope around 1996 (Hollis et al. 2000) which may explain the apparent equatorial molecular ring. No plausible large scale motion was detected for U Ori.

The linear polarization fraction is found to show strong variations between epochs. They are similar for both transitions, although in the jet-like features of $o$ Ceti, the $v=1$ transition shows a somewhat larger $m_{1}$ than the $v=0$ transition. The polarization vectors are found to be mostly tangential to the masing shell, indicating a radial magnetic field.

Several jet-like features seen in the $\mathrm{SiO}$ masers of $o$ Ceti and $\mathrm{R}$ Aqr at several epochs show systematic variations of radial velocity along their length. Due to the observing geometry, observed radial velocities will be predominantly non-radial with respect to the star so are not simple outward accelerations.

The polarization structure of these features also show a preference for the $\mathrm{E}$ vectors to be perpendicular to the direction of the feature; this is interpreted as indicating magnetic fields oriented primarily along the feature. Two possible explanations for this are the magnetic field being dragged by the ionized component of the envelope or the magnetic field controlling the motion of the accelerating material. Several recent $\mathrm{SiO}$ and $\mathrm{H}_{2} \mathrm{O}$ maser observation indicate that the magnetic field will be dynamically important (Herpin et al. 2003; Vlemmings et al. 2005, 2006). Thus, the jet-like structure are likely due to the masing regions being dragged along the magnetic field. Observations of such features sufficiently closely spaced in time to measure proper motions should help understand the nature of these features.

Acknowledgements. W.V. was supported by a Marie Curie Intra-European Fellowship within the 6th European Community Framework Program under contract number MEIF-CT-2005-010393. S.R. acknowledges support from the NSF through research grant AST-0456047.

\section{References}

Bowers, P. F., \& Knapp, G. R. 1988, ApJ, 332, 299

Cotton, W. D., Mennesson, B., Diamond, P. J., et al. 2004, A\&A, 414, 275

Danchi, W. C., Bester, M., Degiacomi, C. G., Greenhill, L. J., \& Townes, C. H. 1994, AJ, 107, 1469

Deguchi, S., \& Watson, W. D. 1990, ApJ, 354, 649

Desmurs, J. F., Bujarrabal, V., Colomer, F., \& Alcolea, J. 2000, A\&A, 360, 189

Diamond, P. J., \& Kemball, A. J. 2003, ApJ, 599, 1372

Diamond, P. J., Kemball, A. J., Junor, W., et al. 1994, ApJ, 430, L61

Elitzur, M. 1991, ApJ, 370, 407

Elitzur, M. 1996, ApJ, 457, 415

ESA. 1997, VizieR Online Data Catalog, 1239, 0

Etoka, S., \& Diamond, P. 2004, MNRAS, 348, 34

Goldreich, P., Keeley, D. A., \& Kwan, J. Y. 1973, ApJ, 179, 111

Herpin, F., Baudry, A., Thum, C., Morris, D., \& Wiesemeyer, H. 2003, in Semaine de l'Astrophysique Francaise, SF2A-2003, 523

Herpin, F., Baudry, A., Thum, C., Morris, D., \& Wiesemeyer, H. 2006 [arXiv: astro-ph/0601098]

Hollis, J. M., Bertram, R., Wagner, R. M., \& Lampland, C. O. 1999, ApJ, 514, 895

Hollis, J. M., Boboltz, D. A., Pedelty, J. A., White, S. M., \& Forster, J. R. 2001, ApJ, 559, L37

Hollis, J. M., Pedelty, J. A., Forster, J. R., et al. 2000, ApJ, 543, L81

Humphreys, E. M. L., Gray, M. D., Yates, J. A., et al. 1996, MNRAS, 282, 1359

Humphreys, E. M. L., Gray, M. D., Yates, J. A., et al. 2002, A\&A, 386, 256

Kemball, A. J., \& Diamond, P. J. 1997, ApJ, 481, L111

Mennesson, B., Perrin, G., Chagnon, G., et al. 2002, ApJ, 579, 446

Nedoluha, G. E., \& Watson, W. D. 1990, ApJ, 354, 660

Nedoluha, G. E., \& Watson, W. D. 1994, ApJ, 423, 394

Pardo, J. R., Alcolea, J., Bujarrabal, V., et al. 2004, A\&A, 424, 145

Perrin, G., Ridgway, S. T., Mennesson, B., et al. 2004, A\&A, 426, 279

Reid, M. J., \& Menten, K. M. 1997, ApJ, 476, 327

Reid, M. J., \& Moran, J. M. 1981, ARA\&A, 19, 231

Soria-Ruiz, R., Alcolea, J., Colomer, F., et al. 2004, A\&A, 426, 131

Vlemmings, W. H. T., Diamond, P. J., \& Imai. 2006, Nature, 440, 58

Vlemmings, W. H. T., Diamond, P. J., \& van Langevelde, H. J. 2002, A\&A, 394 , 589

Vlemmings, W. H. T., van Langevelde, H. J., \& Diamond, P. J. 2005, A\&A, 434, 1029

Yi, J., Booth, R. S., Conway, J. E., \& Diamond, P. J. 2005, A\&A, 432, 531 
W. D. Cotton et al.: Further VLBA observations of SiO masers toward Mira variable stars, Online Material $p 1$

\section{Online Material}


W. D. Cotton et al.: Further VLBA observations of SiO masers toward Mira variable stars, Online Material p 2
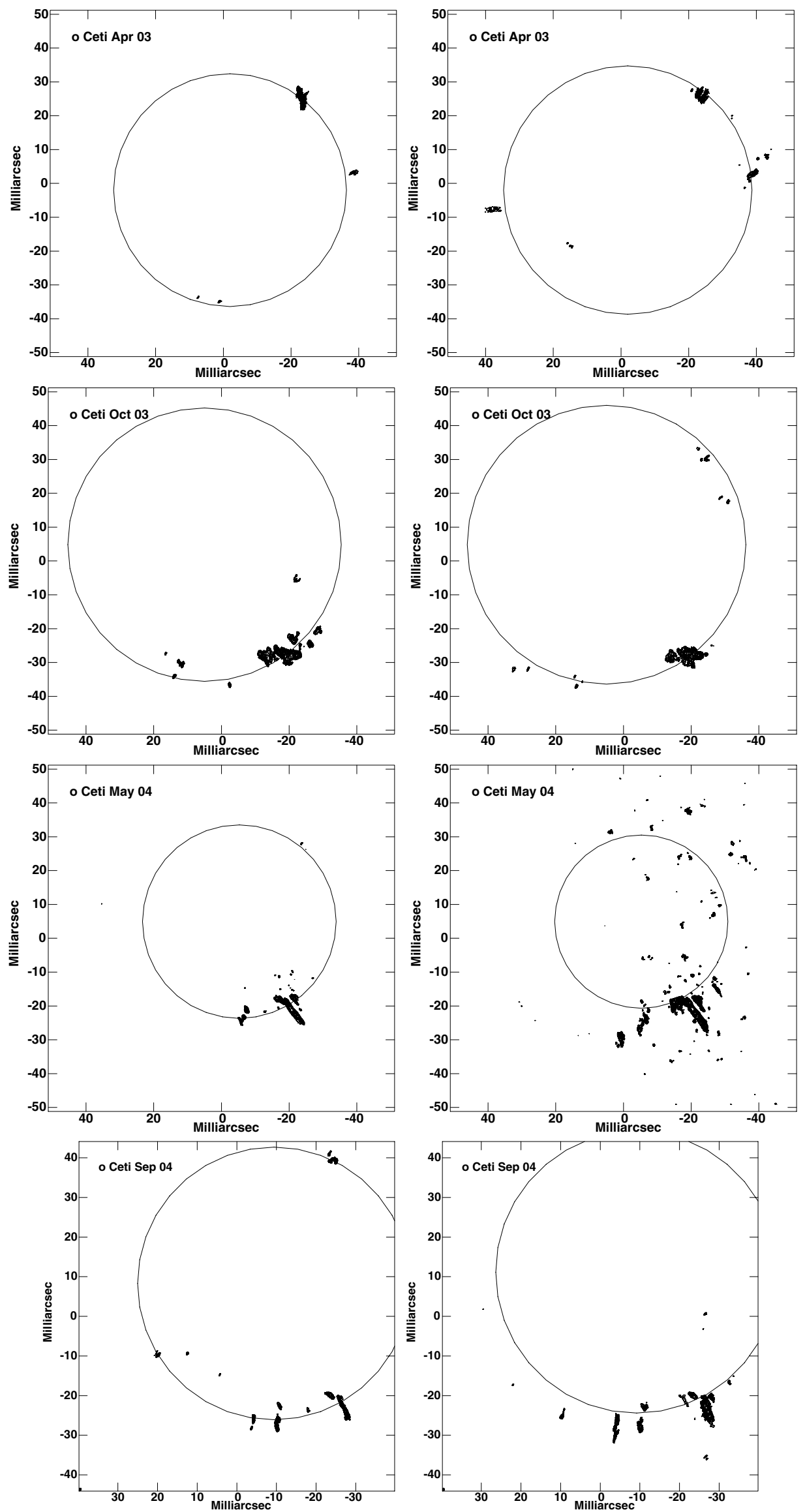

Fig. 1. $o$ Ceti (Mira). Left column is $v=2, J=1-0$ transition of $\mathrm{SiO}$ at $42.8 \mathrm{GHz}$, right column is $v=1, J=1-0$ transition of $\mathrm{SiO}$ at $43.1 \mathrm{GHz}$. Contours of total intensity are shown with a spacing of powers of $\sqrt{2}$. Circles show the fitted rings as given in Table 2 and are centered on the location determined to be the stellar position. 
W. D. Cotton et al.: Further VLBA observations of SiO masers toward Mira variable stars, Online Material p 3
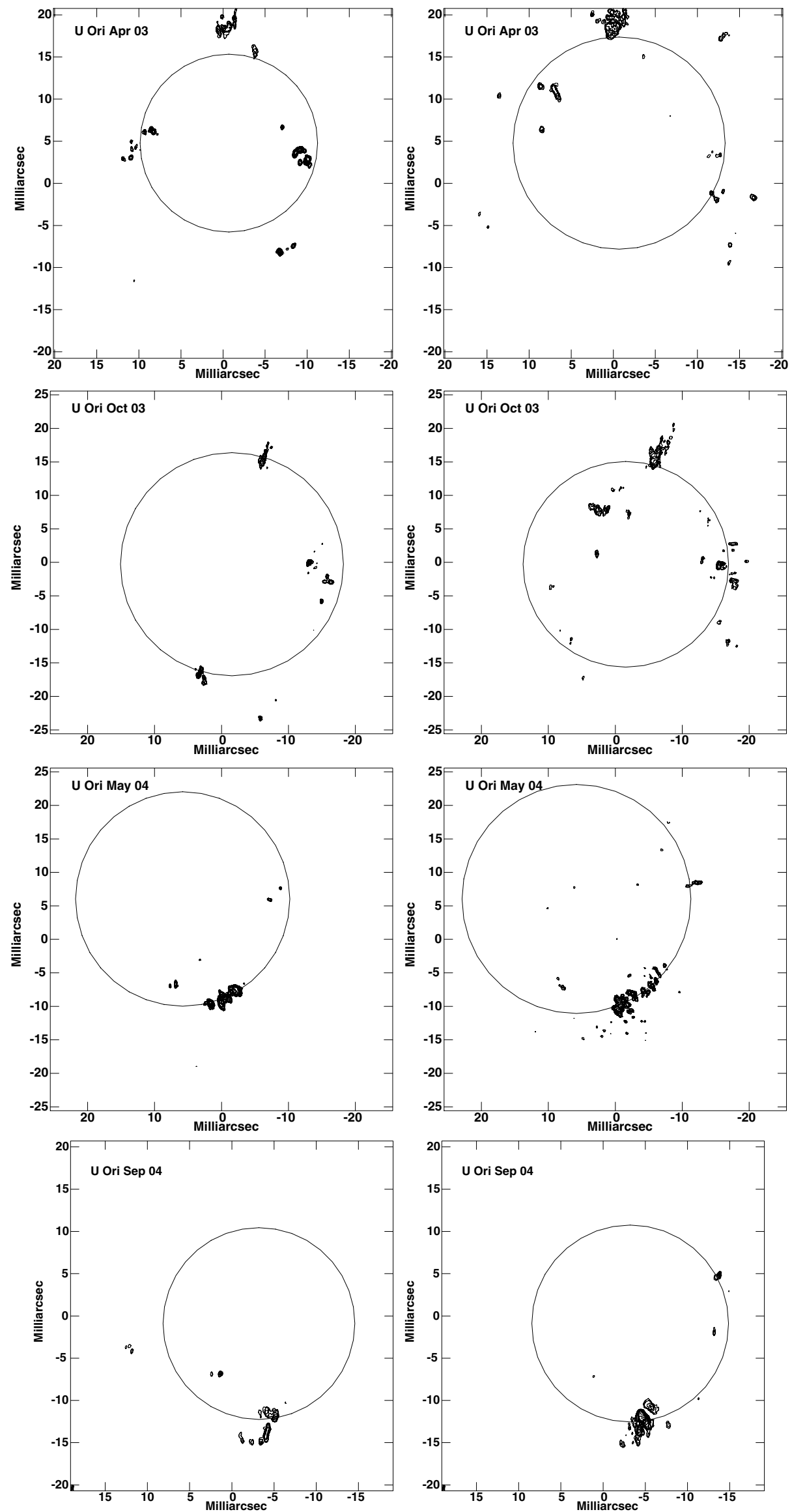

Fig. 2. U Ori. Left column is $v=2, J=1-0$ transition of $\mathrm{SiO}$ at $42.8 \mathrm{GHz}$, right column is $v=1, J=1-0$ transition of $\mathrm{SiO}$ at $43.1 \mathrm{GHz}$. Contours of total intensity are shown with a spacing of powers of $\sqrt{2}$. Circles show the fitted rings as given in Table 2 and are centered on the location determined to be the stellar position. 
W. D. Cotton et al.: Further VLBA observations of SiO masers toward Mira variable stars, Online Material p 4
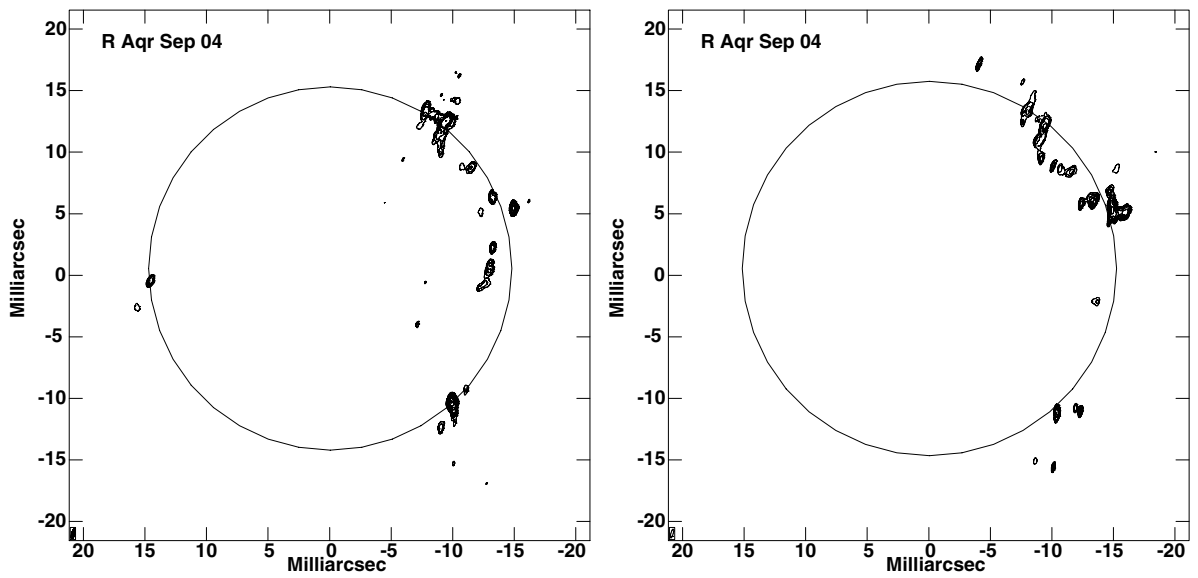

Fig. 3. R Aqr. Left column is $v=2, J=1-0$ transition of $\mathrm{SiO}$ at $42.8 \mathrm{GHz}$, right column is $v=1, J=1-0$ transition of $\mathrm{SiO}$ at $43.1 \mathrm{GHz}$. Contours of total intensity are shown with a spacing of powers of $\sqrt{2}$. Circles show the fitted rings as given in Table 2 and are centered on the location determined to be the stellar position. 
W. D. Cotton et al.: Further VLBA observations of SiO masers toward Mira variable stars, Online Material p 5
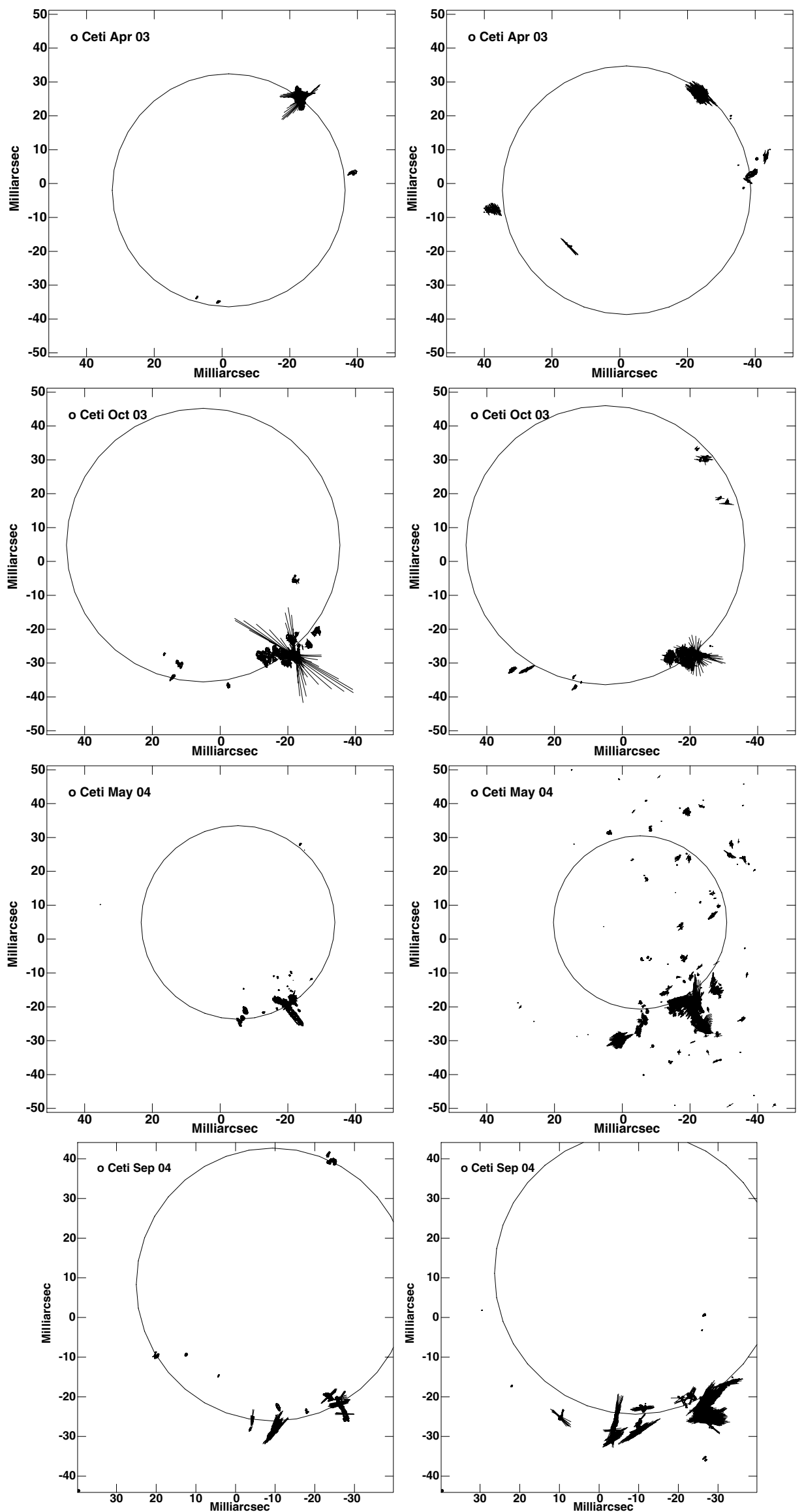

Fig. 1. $o$ Ceti (Mira). Left column is $v=2, J=1-0$ transition of $\mathrm{SiO}$ at $42.8 \mathrm{GHz}$, right column is $v=1, J=1-0$ transition of $\mathrm{SiO}$ at $43.1 \mathrm{GHz}$. Polarization "E" vectors are over-plotted on contours of total intensity; the contours are spaced by powers of $\sqrt{2}$. Circles show the fitted rings as given in Table 2 and are centered on the location determined to be the stellar position. 
W. D. Cotton et al.: Further VLBA observations of SiO masers toward Mira variable stars, Online Material p 6
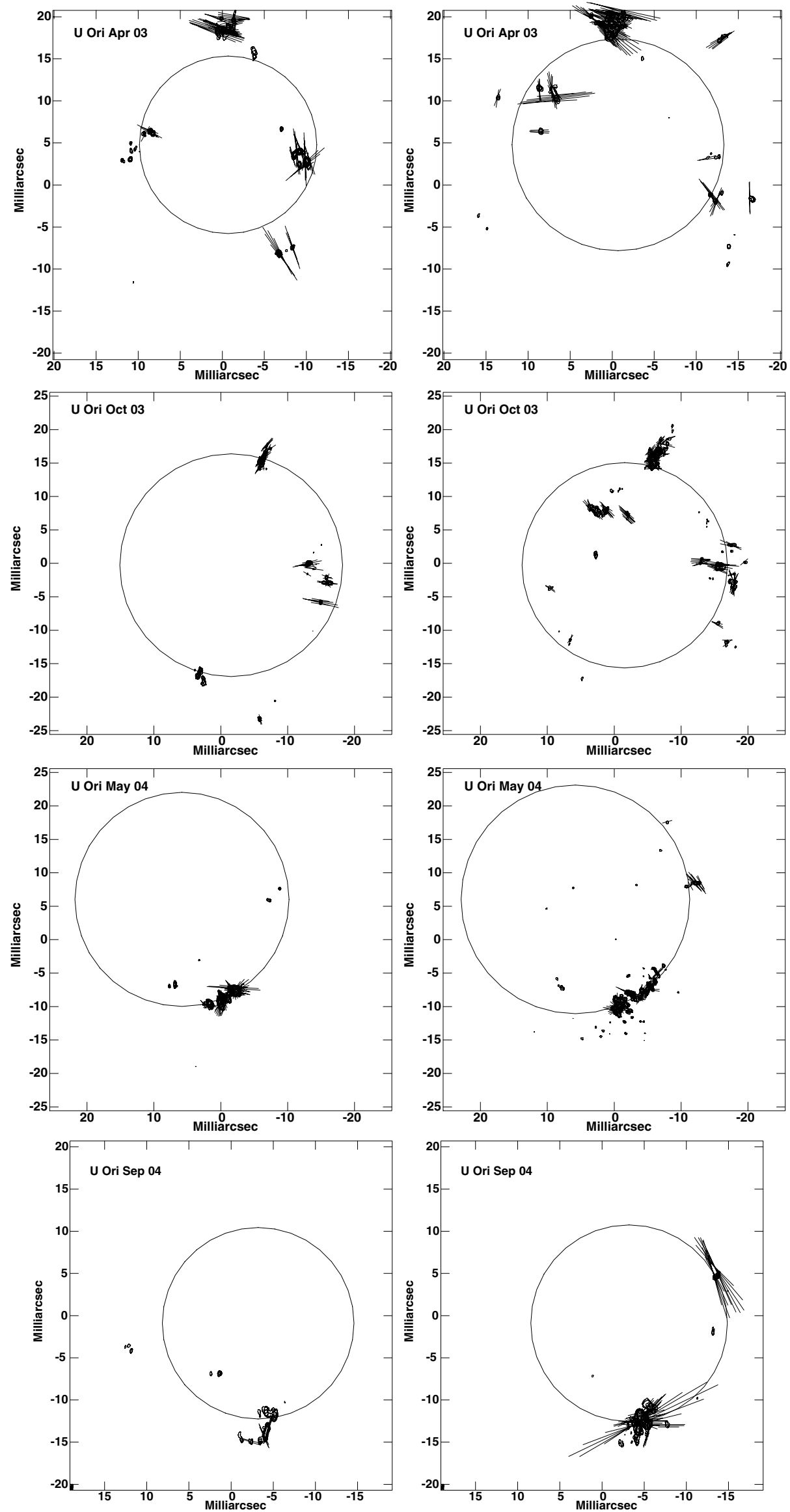

Fig. 2. U Ori. Left column is $v=2, J=1-0$ transition of $\mathrm{SiO}$ at $42.8 \mathrm{GHz}$, right column is $v=1, J=1-0$ transition of $\mathrm{SiO}$ at $43.1 \mathrm{GHz}$. Polarization "E" vectors are over-plotted on contours of total intensity; the contours are spaced by powers of $\sqrt{2}$. Circles show the fitted rings as given in Table 2 and are centered on the location determined to be the stellar position. 
W. D. Cotton et al.: Further VLBA observations of SiO masers toward Mira variable stars, Online Material $p 7$
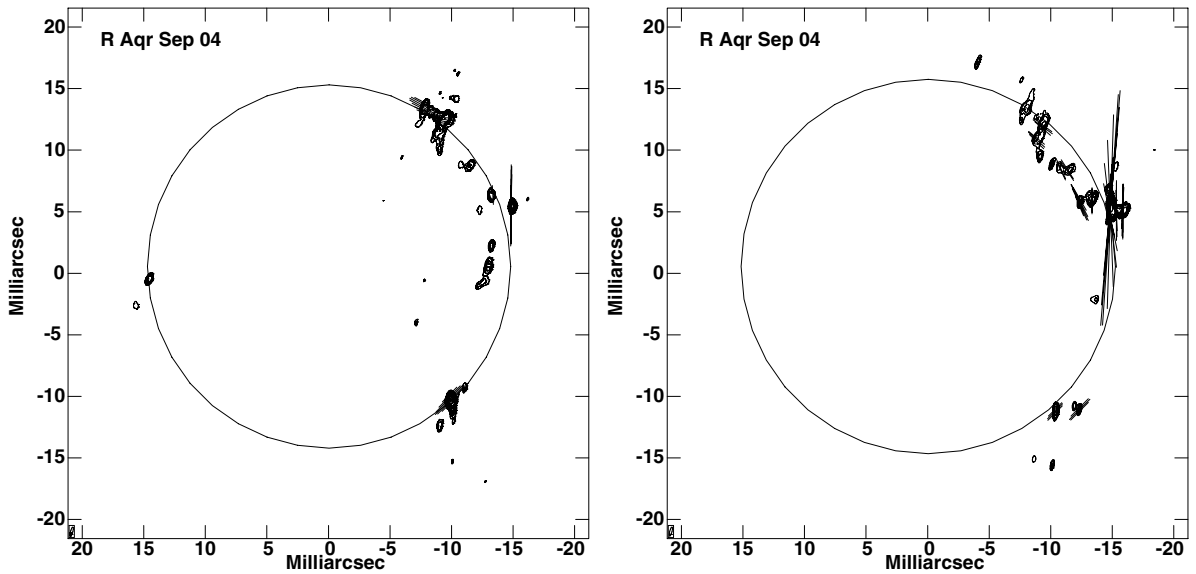

Fig. 3. R Aqr. Left column is $v=2, J=1-0$ transition of $\mathrm{SiO}$ at $42.8 \mathrm{GHz}$, right column is $v=1, J=1-0$ transition of $\mathrm{SiO}$ at $43.1 \mathrm{GHz}$. Polarization "E" vectors are over-plotted on contours of total intensity; the contours are spaced by powers of $\sqrt{2}$. Circles show the fitted rings as given in Table 2 and are centered on the location determined to be the stellar position. 